\title{
OXIDACIÓN DE N-HEPTANO MEDIADA POR EL COMPUESTO DE COORDINACIÓN [Mn $\left(\mathrm{C}_{16} \mathrm{H}_{14} \mathrm{~N}_{2} \mathrm{O}_{2}\right)$ AcO]
}

\author{
N-HEPTANE OXIDATION MEDIATED BY THE \\ COORDINATION COMPOUND [Mn $\left(\mathrm{C}_{16} \mathrm{H}_{14} \mathbf{N}_{2} \mathrm{O}_{2}\right)$ AcO]
}

\author{
Camilo Javier Viasus 1, Raúl Alberto Riveros 2, John Alexander Alzate 3, Ana Esperanza Burgos 4
}

\begin{abstract}
1M.Sc. Química, Químico, Profesor Instructor; Grupo de Investigación en Compuestos de Coordinación y Catálisis - GI3C, Facultad de Ciencia y Tecnología, Universidad de Ciencias Aplicadas y Ambientales, U.D.C.A, Sede Norte, Calle 72 No. 14-20, Bogotá, Colombia. e-mail: caviasus@udca.edu.co. 2Químico, Universidad Nacional de Colombia, Profesor Catedrático, Universidad de Ciencias Aplicadas y Ambientales U.D.C.A. e-mail: jaalzateca@unal.edu.co. ${ }^{3}$ Químico, Universidad Nacional de Colombia. Bogotá, Colombia. e-mail: ariverosr@unal.edu.co. ${ }^{4}$ Dr. Sc. Química, Profesor Asociado, Laboratorio de Compuestos de Coordinación y Bioinorgánica, Universidad Nacional de Colombia, Carrera 30 No. 45-03, Bogotá. Colombia. e-mail: aeburgosc@unal.edu.co.
\end{abstract}

Rev. U.D.C.A Act. E Div. Cient. 14(2): 141 - 149, 2011

\section{RESUMEN}

La cantidad de hidrocarburos que son producidos en la actualidad por la industria petrolera continúan en incremento, causando gran contaminación ambiental. La reacción lograda en este trabajo es una alternativa a la recuperación de residuos hidrocarbonados industriales. En este trabajo, se presenta la oxidación de n-heptano, mediada por el compuesto de coordinación [ $\left.\mathrm{Mn}\left(\mathrm{C}_{16} \mathrm{H}_{14} \mathrm{~N}_{2} \mathrm{O}_{2}\right) \mathrm{AcO}\right]$. Los productos más significativos de la reacción fueron alcoholes (n-butanol) y cetonas de siete carbonos (2-heptanona, 3-heptanona y 4-heptanona, principalmente). La oxidación de n-heptano se ve favorecida hacia la formación de n-butanol, en medio acuoso y, a la formación de cetonas, en medio no acuoso. Los productos de la oxidación fueron identificados mediante CG-EM.

Palabras clave: Oxidación, hidrocarburos, [Mn(salen)AcO], recuperación de residuos.

\section{SUMMARY}

The amounts of hydrocarbons that are produced today by the oil industry continue to increase causing great environmental pollution. The reaction present here is an alternative to waste hydrocarbons recovery. In this work it present the n-heptane oxidation mediated by the coordination compound $\left[\mathrm{Mn}\left(\mathrm{C}_{16} \mathrm{H}_{14} \mathrm{~N}_{2} \mathrm{O}_{2}\right) \mathrm{AcO}\right]$. The most significant reaction products were alcohol (n-buthanol) and seven carbons ketones (mainly 2-heptanone, 3-heptanone and 4-heptanone). The n-hepthane oxidation is favored toward the formation to n-butanol in aqueous medium and ketone formation in solvent without water. The oxidation products were identified by GC-MS

Key words: Oxidation, hydrocarbons, [Mn(salen)AcO], waste recovery.

\section{INTRODUCCIÓN}

La oxidación de hidrocarburos, mediante la activación del enlace $\mathrm{CH}$ para obtener compuestos diferentes a los convencionales, como $\mathrm{CO}_{2}$, consiste en la preservación, la reutilización y la buena disposición de compuestos residuales, provenientes de procesos industriales, como petroquímica, gas natural y tratamiento de crudos. Este procedimiento hace que sea valiosa la incorporación de funciones oxigenadas a dichas cadenas (Dyker, 2005; Olah \& Molnár, 2003; Shilov E Shul’Pin, 2000).

Robert H. Crabtree, en el 2004, muestra cómo la activación del enlace $\mathrm{C}$-H en alcanos y su funcionalización ha sido muy estudiada y reportada. De manera general, es ampliamente aceptado que la funcionalización de alcanos se puede realizar por dos mecanismos: el primero, completamente organometálico y, el segundo, mediante química de coordinación; en los dos casos, existe una interacción directa con el sustrato por el átomo de carbono o imitando la actividad del citocromo P450 y así poder eliminar las sustancias tóxicas poco polares en el cuerpo humano para hacerlas más polares, solubles en agua y que puedan ser excretadas por la orina (Costas, 2011). 
El uso de compuestos de coordinación para realizar oxidaciones, más específicamente epoxidaciones, no es un tema muy novedoso. Los primeros ensayos muestran que los compuestos de coordinación con anillos pirrólicos presentan gran actividad para las epoxidaciones enantioselectivas de alquenos (Jacobsen et al. 1994; 1997; Jacobsen, 1995); esta actividad se atribuye a la gran similitud estructural del citocromo C (Reacción (1)) con las metaloporfirinas. Los ensayos realizados muestran que el uso de compuestos de coordinación aceleran las epoxidaciones para la formación de epóxidos y alcoholes, siempre y cuando se realice un tratamiento ácido, posterior a la formación del epóxido (Shahram et al. 2010; Costas, 2011).
La oxidación de los hidrocarburos insaturados y saturados cíclicos ocurre a bajas temperaturas en presencia de oxidantes, como PhIO y $\mathrm{H}_{2} \mathrm{O}_{2}$ (Palucki et al. 1992; 1995), utilizando compuestos de coordinación simples, tales como [Mn(salen)] y sus derivados.

La modificación química de hidrocarburos parte de la alteración de polímeros, siendo un método de gran importancia para la preparación de nuevos materiales con diversas propiedades; por ejemplo, en la modificación post-polimerización de poli-dienos, se han utilizado reacciones, como hidrogenación, epoxidación, clorosilación, hidroboración y adiciones de carbenos. Estas reacciones aportan gran es-

$$
\mathrm{C}-\mathrm{H} / \mathrm{C}=\mathrm{C}+\mathrm{O}_{2}+2 \mathrm{H}^{+}+2 \mathrm{e}^{-} \stackrel{\mathrm{P} 450}{\longrightarrow} \mathrm{C}-\mathrm{OH} / \stackrel{\mathrm{O}}{\mathrm{C}-\mathrm{C}}+\mathrm{H}_{2} \mathrm{O}
$$

\section{MATERIALES Y MÉTODOS}

tabilidad a la transformación de dobles enlaces carbonocarbono y la adición de la funcionalización a lo largo de los dobles enlaces de la estructura (Boaen $\mathcal{E}$ Hillmyer, 2003).

Uno de los inconvenientes de las funcionalizaciones es la ausencia de grupos funcionales reactivos; sin embargo, la búsqueda de rutas para funcionalizar materiales, adicionando un valor agregado, continúa, incluyendo las modificaciones parciales que se realizan a pigmentos y otro tipo de macromoléculas, que permiten generar otras propiedades fisicoquímicas o mejorar las que ya poseen, a través de los nuevos grupos funcionales adicionados (Boaen E Hillmyer, 2003).

La funcionalización de alcanos de bajo peso molecular, mediante la química de coordinación, ha sido bastante explorada, a través de los años, logrando funcionalizar, satisfactoriamente, una gran cantidad de alcanos, por el uso de compuestos porfirinicos y con bases de Schiff unidos a sílice con manganeso(III), como ion central (Valiollah et al. 2006) y otros metales, como rutenio(II) (Mehdi et al. 2009) y hierro(III) (Sun Chang et al. 2011).

Se han aplicado sistemas porfirínicos más complicados, demostrando que la oxidación de n-heptano es posible hacia la formación de alcoholes y de cetonas (Costas, 2011). En este trabajo, se utilizó un sistema simple y diferente a los publicados hasta el momento: el compuesto de coordinación [Mn(Salen) AcOl $\left(\right.$ Salen $=\mathrm{N}^{\prime} \mathrm{N}^{\prime}$-bis-salicilaldehidoetilendiammin, $\mathrm{C}_{16} \mathrm{H}$ $\left.{ }_{14} \mathrm{~N}_{2} \mathrm{O}_{2}\right)$. Con este complejo de coordinación, se analiza la posibilidad de realizar la activación de enlaces $\mathrm{C}$-H de alcanos lineales y ramificados de bajo peso molecular, como base para la recuperación de residuos hidrocarbonados.
Preparación del ligante Salen: El ligante salen se sintetizó, mezclando salicilaldehído y etilendiamina, en relación molar 2:1, en $20 \mathrm{~mL}$ de acetonitrilo seco y bajo reflujo, durante dos horas. Al enfriar el sistema a temperatura ambiente fueron obtenidos cristales amarillos brillantes, filtrados y secados al vacío, durante 24 horas (Shahram et al. 2010; Pecoraro E Butler, 1986). El sólido obtenido se caracterizó, mediante las técnicas espectroscópicas UV-vis, FT-IR, 1H RMN y DEPT 135. El rendimiento de la reacción fue del 97,6\%.

Preparación del compuesto [Mn(salen)AcO]: El compuesto de coordinación $\left[\mathrm{Mn}\left(\mathrm{C}_{16} \mathrm{H}_{14} \mathrm{~N}_{2} \mathrm{O}_{2}\right) \mathrm{AcO}\right]$ fue sintetizado, mezclando el ligante salen con acetato de manganeso(II) anhidro, en 15mL acetonitrilo seco, en relación molar 1:1. La mezcla fue puesta en reflujo durante dos horas (Shahram et. al. 2010; Pecoraro E Butler, 1986). Se obtuvo un sólido amarillo oscuro, secado al vacío, durante 24 horas e identificado mediante las técnicas espectroscópicas UV-Vis y FT-IR. El rendimiento de la reacción fue del 87,3\%. Las reacciones de la preparación del ligante salen y el compuesto [Mn(salen)AcO], se presentan en la figura 1.

Oxidación de n-heptano: La reacción de oxidación de nheptano, se realizó de acuerdo con el procedimiento reportado por Boaen $\mathcal{E}$ Hillmyer (2003), en cuanto a los reactivos y a las cantidades molares, pero en este trabajo, se utilizó el compuesto de coordinación $\left[\mathrm{Mn}\left(\mathrm{C}_{16} \mathrm{H}_{14} \mathrm{~N}_{2} \mathrm{O}_{2}\right) \mathrm{AcO}\right]$, como catalizador. La reacción de oxidación, se efectuó en acetonitrilo y $\mathrm{CH}_{2} \mathrm{Cl}_{2}$, como disolventes; $\mathrm{NaIO}_{4}$, como agente oxi- 
2<smiles>O=Cc1ccccc1O</smiles><smiles>NCCN</smiles><smiles>OC1=CC=[C+]C=C1/C=N/CC/N=C/c1ccccc1O</smiles>

$\mathrm{ACN}$

Reflujo 3h

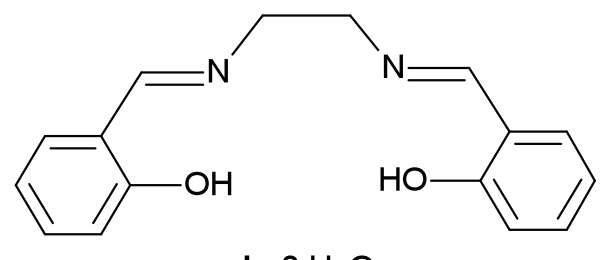

$+2 \mathrm{H}_{2} \mathrm{O}$
$\mathrm{ACN}$<smiles>[R20]O[R6]([H])([H])[H]</smiles>

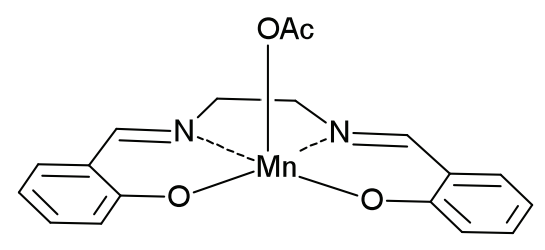

Figura 1. Reacción para la obtención del ligante salen (1) y de la obtención del complejo $\left[\mathrm{Mn}\left(\mathrm{C}_{16} \mathrm{H}_{14} \mathrm{~N}_{2} \mathrm{O}_{2}\right)\right.$ AcO] (2) en acetonitrilo, respectivamente.

dante; imidazol, para incrementar la densidad electrónica sobre el centro metálico, BDTAC (cloruro de bencildimetiltetradecilamonio), como agente de transferencia de fase y se suprimió el medio acuoso, para evitar la descomposición del compuesto de coordinación; la reacción, se llevó a cabo en un recipiente tipo Parr ${ }^{\circledR}$, cerrado herméticamente, a $60^{\circ} \mathrm{C}$ en autoclave, durante cuatro horas. Los compuestos obtenidos de la reacción de oxidación de n-heptano fueron caracterizados, mediante CG-EM (reacción 2).

\section{RESULTADOS Y DISCUSIÓN}

El ligante salen se identificó mediante espectroscopia FT-IR (Figura 2), que registra la vibración más importante, que es la formación del doble enlace de la imina, a $1627 \mathrm{~cm}^{-1}$. Así mismo, se presentan las vibraciones para $-\mathrm{CH}_{2^{-}}$, a $2924 \mathrm{~cm}^{-1}$ y C $=\mathrm{C}$ aromático, a 1496 y $1458 \mathrm{~cm}^{-1}$, entre otras. El espectro de ${ }_{1}^{1} \mathrm{H}-\mathrm{NMR}$, para el ligante salen, se muestra en la figura 3a. Los

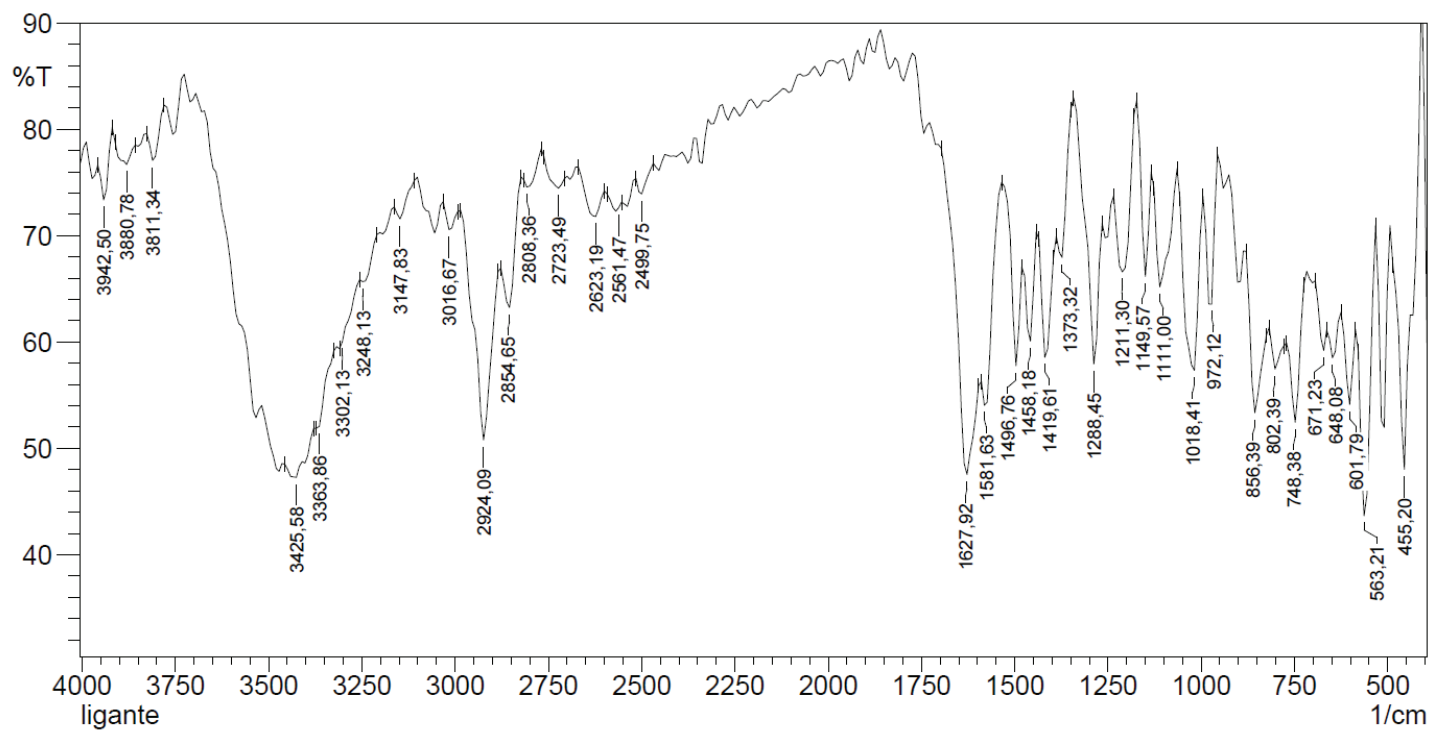

Figura 2. Espectro IR para el ligante salen. 
a)
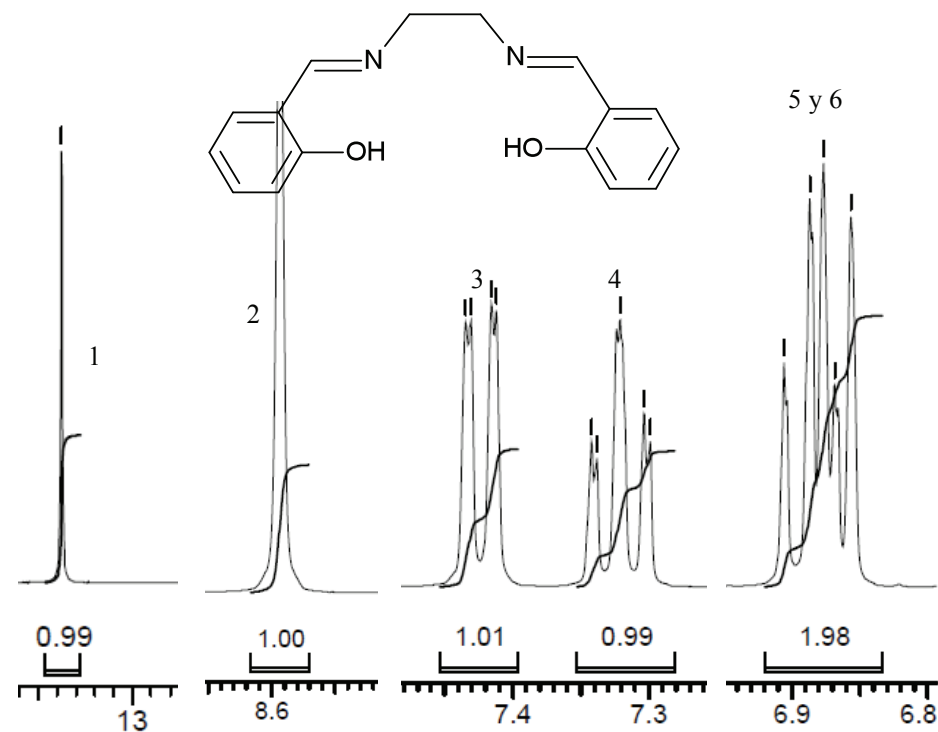

$7.4+7.3$

6.9

6.8

7

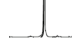

b)
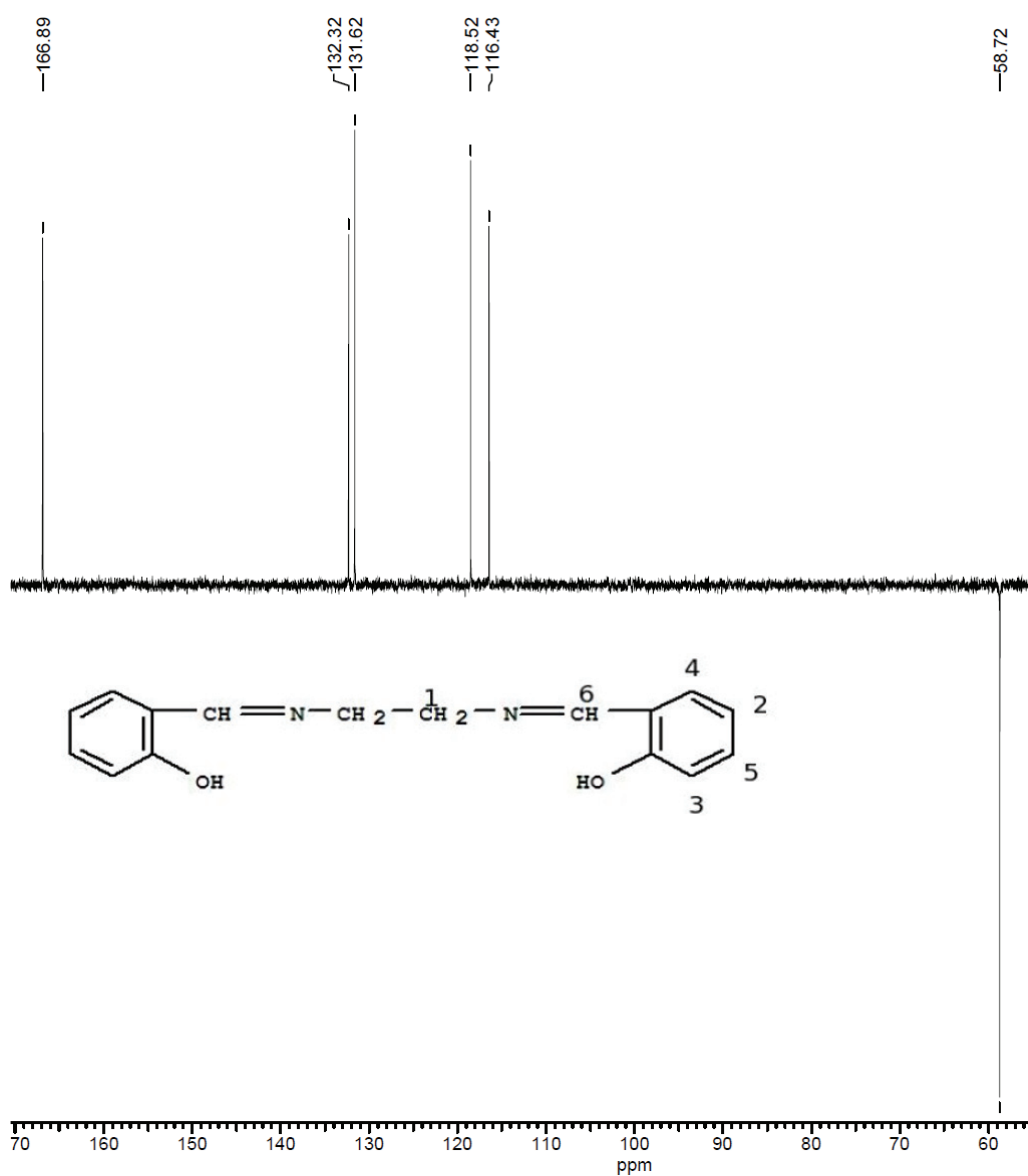

Figura 3. a) Espectro expandido y por secciones de ${ }^{1} \mathrm{H}-\mathrm{NMR}$ para el ligante salen; b) espectro DEPT 135 para el ligante salen. 
desplazamientos químicos $\partial 1570 \mathrm{MHz}$, que integra para dos protones $\left(-\mathrm{CH}_{2}-\right)$, en 2743 y $2763 \mathrm{MHz}$, presenta un doblete y un triple superpuestos, que corresponden a la integración de los dos protones 5 y 6 C-H aromático; un triplete en $2929 \mathrm{MHz}$, asignado a la posición 4; un doblete en $2973 \mathrm{MHz}$, designado a la posición 3; un singlete en $3438 \mathrm{MHz}$, estipulado $\mathrm{a}=\mathrm{CH}$ de la imina, posición 2 y, finalmente un singlete en $5352 \mathrm{MHz}$, asignado para el hidrógeno en la posición 1. Estas últimas cuatro posiciones integran, por un protón, cada una, para un total de 8 protones; por la simetría de la molécula, el total son 16 protones. Estas mismas posiciones, se utilizaron para identificar el espectro DEPT135.

El espectro DEPT135 (Figura 3b) para el ligante salen presenta un desplazamiento en 58,72ppm, se asigna a -
$\mathrm{CH}_{2}$, posición 7; el desplazamiento en $116,43,118,52$, 131,62 y $132,32 \mathrm{ppm}$, se asignan a las posiciones $2,3,4$ y 5 , respectivamente y, finalmente, el desplazamiento en 166,89ppm, asignado a $=\mathrm{CH}$ de la imina. Los espectros UVvis para el ligante salen y para el compuesto de coordinación [Mn(Salen)AcO], se presentan en la figura 4, donde el efecto hipsocrómico de la coordinación del ion metálico con el ligante salen, se hace evidente. La tabla 1 registra las máximas absorciones para el ligante y para el $\left[\mathrm{Mn}\left(\mathrm{C}_{16} \mathrm{H}_{14} \mathrm{~N}_{2} \mathrm{O}_{2}\right) \mathrm{AcO}\right]$.

El compuesto de coordinación, se identificó mediante espectroscopia UV-vis e IR. El corrimiento en la vibración del enlace $\mathrm{C}=\mathrm{N}$ a $1635 \mathrm{~cm}^{-1}$ que indica la formación del enlace $\mathrm{Mn}-\mathrm{N}$ junto a las vibraciones en 455 y $425 \mathrm{~cm}^{-1}$, corresponde al enlace $\mathrm{Mn}-\mathrm{O}$ y $\mathrm{Mn}-\mathrm{N}$.

Tabla 1. Máximos de absorción para el ligante salen y para el complejo [Mn(salen)AcO].

\begin{tabular}{|c|c|c|c|}
\hline \multirow{5}{*}{ Salen } & Longitud de onda & \multirow{5}{*}{ [Mn(salen)AcO] } & Longitud de onda \\
\hline & 215 & & 210 \\
\hline & 255 & & 240 \\
\hline & 320 & & 310 \\
\hline & 405 & & 395 а 400 \\
\hline
\end{tabular}

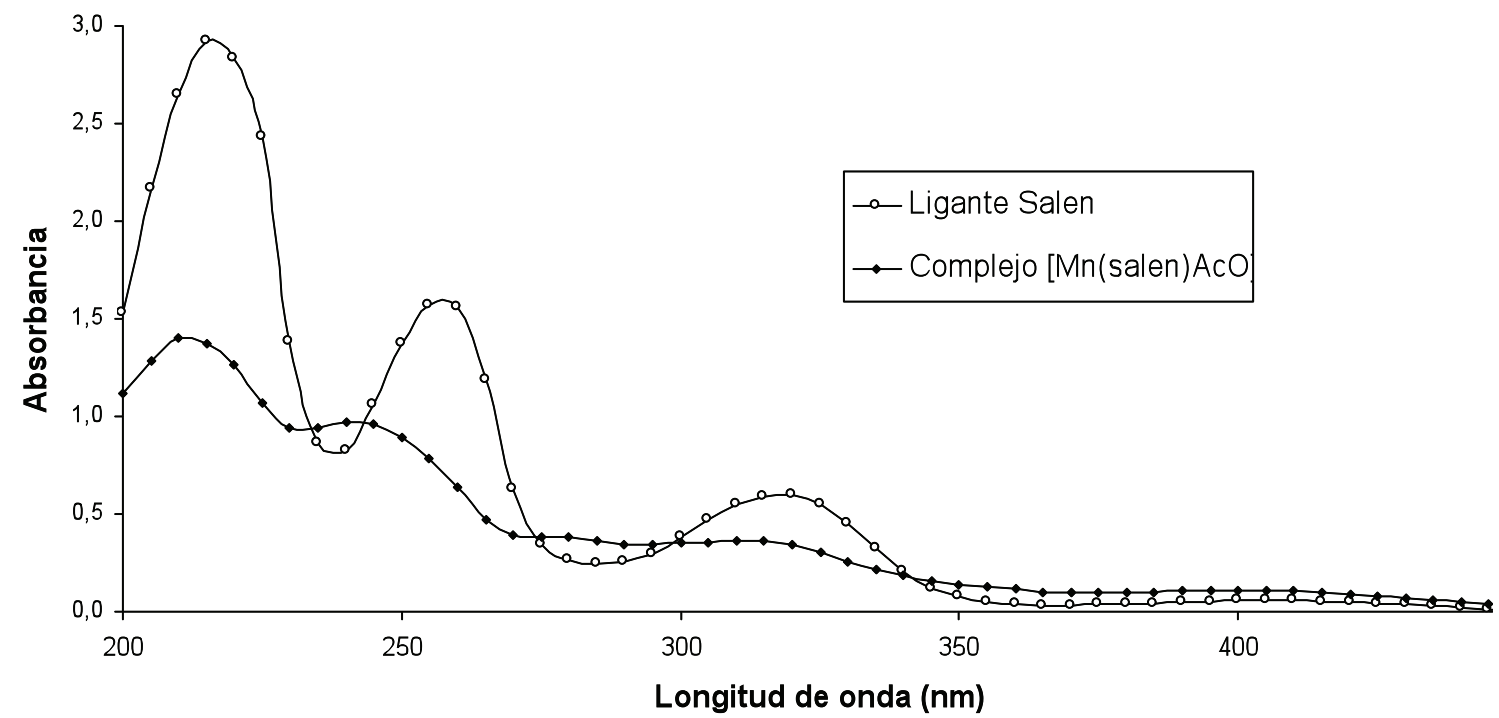

Figura 4. Espectro UV-vis del ligante Salen y del compuesto de coordinación [Mn(salen)AcO] en ACN. 
La oxidación de n-heptano, se realiza de acuerdo a la

reacción (2).

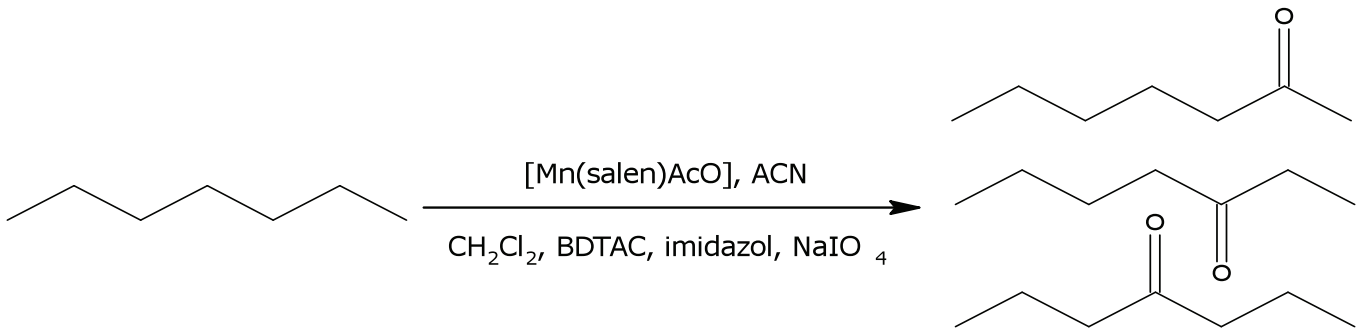

El medio de reacción resultó ser selectivo a la formación de cetonas de siete carbonos con bajos rendimientos. En publicaciones presentadas con anterioridad, se obtienen no solamente cetonas, también sus respectivos alcoholes, pero en cantidades menores (Valiollah et. al. 2006) y para sistemas más complejos. Hay que tener en cuenta que los sistemas presentados en literatura, anteriores a este trabajo, han utilizado como sustratos compuestos que, de alguna manera, no presentan linealidad en la cadena hidrocarbonada saturada, es decir, normalmente son complejos cíclicos saturados o sustituidos (Palucki et. al. 1992; 1995). La espectrometría de masas mostró que los compuestos principales obtenidos durante la oxidación de n-heptano aplicando el compuesto de coordinación sintetizado son 2-heptanona, 3-heptanona y 4 heptanona.

Aunque los compuestos obtenidos principalmente fueron cetonas, se presentaron trazas de compuestos menos oxidados, como 2-heptanol y 3-heptanol (Figura 5d y 5e). El resultado de la oxidación completa, se debe al agente donador de oxígenos presente en la reacción, como el $\mathrm{NaIO}_{4}$, así este sistema propuesto para la oxidación de n-heptano resulta ser útil y más sencillo de elaborar que el realizado por Costas (2011), para la oxidación del mismo sustrato.

Debido a que los pesos moleculares son los mismos para cada una de las cetonas presentes, en los rompimientos se evidencia la presencia de cada uno de los compuestos mencionados; para los tres casos, el pico de ion molecular fue de $114 \mathrm{~m} / \mathrm{z}\left(\mathrm{C}_{7} \mathrm{H}_{14} \mathrm{O}^{+}\right.$.). Para el compuesto 4-heptanona, se presenta una pérdida de $43 \mathrm{~m} / z$, correspondiente a ${ }^{+} \mathrm{CH}_{2} \mathrm{CH}_{2} \mathrm{CH}_{3}$, seguida por una pérdida de $28 \mathrm{~m} / \mathrm{z}$, correspondiente a CO; el pico padre se encuentra en $71 \mathrm{~m} / \mathrm{z}$, que corresponde $\mathrm{a}_{3} \mathrm{H}_{7} \mathrm{CO}+$. Para el compuesto 3-heptanona, se muestra una pérdida inicial de $29 \mathrm{~m} / \mathrm{z}$, correspondiente a $\mathrm{CH}_{2} \mathrm{CH}_{3}$ y con pico padre de $54 \mathrm{~m} / \mathrm{z}$, correspondiente a $\mathrm{CH}_{3} \mathrm{CH}_{2} \mathrm{CO}^{+}$. Para el compuesto 2-heptanona, se presenta una pérdida de $43 \mathrm{~m} / \mathrm{z}$, correspondientes a $\mathrm{COCH}_{3}$, seguido por una pérdida de $13 \mathrm{~m} / \mathrm{z}$, que corresponde a $\mathrm{CH}$, luego una pérdida de $15 \mathrm{~m} / \mathrm{z}$, correspondiente a $\mathrm{CH}_{3}$ y con pico padre de $43 \mathrm{~m} / \mathrm{z}$, correspondiente a $\mathrm{CH}_{3} \mathrm{CO}^{+}$. Los espectros de masas para 2-heptanona, 3-heptanona y 4-heptanona, se indican en las figuras $5 a, 5 b$ y $5 c$, respectivamente.

Se encontró que al utilizar agua en el medio de reacción, en lugar de ACN, favorece la formación de n-butanol (Figura 5f) y promueve la descomposición de [Mn(salen)AcO].

La conversión total del hidrocarburo utilizado fue del $19 \%$, aproximadamente, en cuatro horas de reacción, en donde se encuentran las cetonas de siete carbonos, alcoholes y la transformación de n-heptano a isómeros, como 2-metilhexano, 3-metilhexano, metilciclohexano y etilciclohexano. Esta última reacción de isomerización, para los compuestos de siete carbonos, ocurre sin la presencia del catalizador, utilizado en este trabajo, pero al usar el catalizador, la reacción se hace selectiva a la isomerización a metilciclohexano. De aquí la importancia en el uso del catalizador en la transformación de hidrocarburos saturados de cadena lineal.

Se propone la utilización de este tipo de sistemas con manganeso(III) para la transformación de hidrocarburos de bajo peso molecular, que se obtienen como residuos en las empresas petroleras. Así, se puede dar un valor agregado a los residuos para poder reutilizarlos en diferentes aplicaciones industriales, lo que generaría, sin lugar a dudas, un menor impacto ambiental.

En conclusión, se sintetizó e identificó un sistema sencillo como el compuesto de coordinación [Mn(Salen)AcO], que resultó ser útil para obtener cetonas, a partir de n-heptano. Aunque los resultados logrados en cuanto a la obtención de compuestos oxidados provenientes de hidrocarburos 


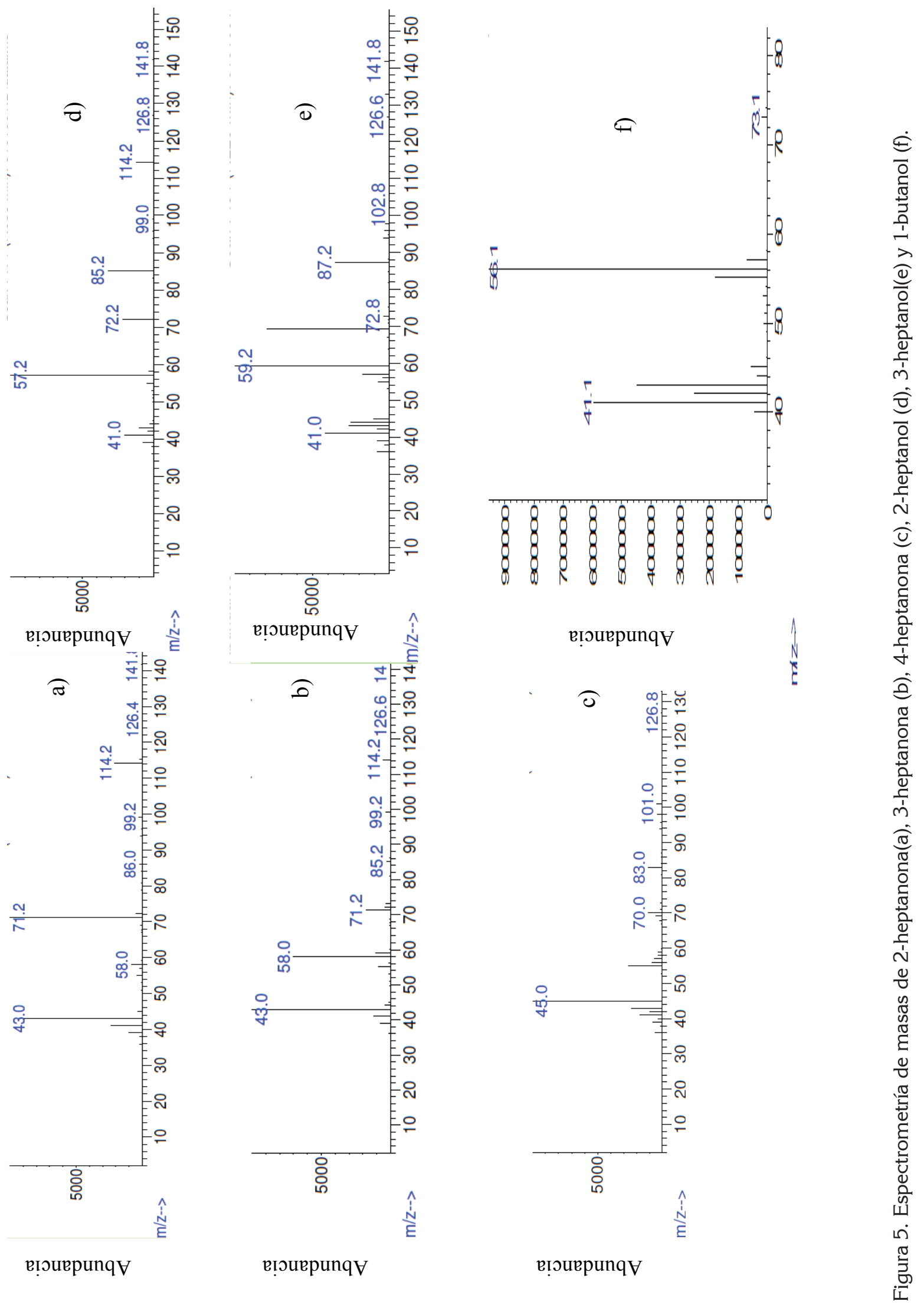


lineales, ya sean alcoholes o cetonas, son inferiores en rendimiento, comparado con el trabajo realizado por Costas (2011). Es cierto que el sistema aquí trabajado es más sencillo y las aplicaciones a la fecha que se han reportado para este tipo de compuestos de coordinación, se limitaba únicamente a la epoxidación de alquenos y a oxidaciones en hidrocarburos cíclicos que son más reactivos que los lineales (Jacobsen, 1995; Jacobsen et al. 1994) y en sistemas a bajas temperaturas (Palucki et al. 1995). Este sistema, se puede emplear en la transformación de hidrocarburos de bajo peso molecular para el mismo fin, lo que reduciría el impacto ambiental que produce normalmente la liberación de estos desechos.

Agradecimientos: Al laboratorio de Macromoléculas, por la toma de los espectros IR. Al laboratorio de Compuestos de Coordinación y Bioinorgánica en la Universidad Nacional de Colombia, Sede Bogotá. A Cheyron Castellanos, por el apoyo brindado en el desarrollo de este proyecto. Conflicto de intereses: Este escrito fue preparado y revisado con la participación de todos los autores, quienes declaramos que no existe ningún conflicto de intereses que ponga en riesgo la validez de los resultados presentados. Financiación: Este estudio fue financiado por la Universidad de Ciencias Aplicadas y Ambientales U.D.C.A, Vicerrectoría de Investigaciones.

\section{BIBLIOGRAFÍA}

1. BOAEN, N.K.; HILLMYER, M.A. 2003. Selective and mild oxyfunctionalization of model polyolefins. Macromolecules, 36(19):7027-7034.

2. COSTAS, M. 2011. Selective C-H oxidation catalyzed by metalloporphyrins. Chem Coord. Rev. In press. Disponible desde Internet en: http://www.sciencedirect. com/science/article/pii/S0010854511001780(con acceso 06/07/11).

3. CRABTREE, R.H. 2004. Organometallic alkane CH activation. J. Organometallic Chem. 689:4083-4091.

4. DYKER, G. 2005. Handbook of $\mathrm{C}-\mathrm{H}$ transformation, Wiley-VCH, Weinheim: p.11-24 y 497-524.

5. JACOBSEN, E.N.; KAKIUCHI, F.; KONSLER, R.G.; LARROW, J.F.; TOKUNAGA, M. 1997. Enantioselective catalytic ring opening of epoxides with carboxylic acids. Tetrahedron Letters. 38(5):773-776.
6. JACOBSEN, E.N. 1995. Transition metal-catalyzed oxidations: asymmetric epoxidation. En: Edward, W.A.; Stone, F.G.A; Geoffrey, W. (eds). Comprehensive organometallic chemistry II. Oxford, Elsevier.p. 10971135.

7. JACOBSEN, E.N.; DENG, L.; FURUKAWA, Y.; MARTÍNEZ, L.E. 1994. Enantioselective catalytic epoxidation of cinnamate esters. Tetrahedron, 50(15):4323-4334.

8. MEHDI, H.; MAJID, M.; IRAN, S.; VALIOLLAH, M.; SHAHRAM, T.; IRAJ, M.; HADI, K. 2009. Ru(salophen)Cl supported on polystyrene-bound imidazole: an efficient and robust heterogeneous catalyst for epoxidation of alkenes with sodium periodate. Applied Catalysis A: General. 370: 66-71.

9. OLAH, G.A.; MOLNÁR, Á. 2003. Hydrocarbon chemistry, 2nd ed., Wiley, New York. P.427-529.

10. PALUCKI, M.; HANSON, P.; JACOBSEN, E.N. 1992. Asymmetric oxidation of sulfides with $\mathrm{H}_{2} \mathrm{O}_{2}$ catalyzed by $\mathrm{Mn}(\mathrm{III})($ Salen) complexes. Tetrahedron Letters. 33(47):7111-7114.

11. PALUCKI, M.; MCCORMICK, G.J.; JACOBSEN, E.N. 1995. Low temperature asymmetric epoxidation of unfunctionalized olefins catalyzed by $\mathrm{Mn}$ (III)(salen) complexes. Tetrahedron Letters. 36(31):5457-5460.

12. PECORARO, V.L.; BUTLER, W.M. 1986. Structure of $\mathrm{N}, \mathrm{N}$ '-ethylenebis(salicylideneiminato)manganese(III) chloride acetonitrile solvate. Acta Crystallography. C42:1151-1154.

13. SHAHRAM, T.; MOGHADAM, M.; MIRKHANI, V.; MOHAMMADPOOR-BALTORK, I.; SAEEDI, M.S. 2010. Efficient epoxidation of alkenes with sodium periodate catalyzed by reusable manganese(III) salophen supported on multi-wall carbon nanotubes. Applied Catalysis A: General. 381:233-241.

14. SHILOV, A.E.; SHUL'PIN, G.B. 2000. Activation and catalytic reactions of saturated hydrocarbons in the presence of metal complexes. Springer-Verlag, Boston. p.8-19 y $127-187$.

15. SUN, C-L.; LI, B.J.; SHI. Z-J. 2011. Direct C-H Transformation via Iron Catalysis. Chemical. Review. 111(3): 1293-314. 
16. VALIOLLAH, M.; MAJID, M.; SHAHRAM, T.; BAHRAM, B. 2006. Silica-bound imidazole as a heterogeneous axial ligand for $\mathrm{Mn}$ (salophen)Cl: efficient, recoverable and recyclable catalyst for epoxidation of alkenes and hydroxylation of alkanes with sodium periodate. Applied Catalysis A: General, 313:122-129.

Recibido: Septiembre 7 de 2011

Aprobado: Noviembre 6 de 2011 